\title{
Nanomaterials Interaction with Cell Membranes: Computer Simulation Studies
}

\author{
Alexey A. Tsukanov and Olga Vasiljeva
}

\begin{abstract}
This chapter provides a brief review of computer simulation studies on the interaction of nanomaterials with biomembranes. The interest in this area is governed by the variety of possible biomedical applications of nanoparticles and nanomaterials as well as by the importance of understanding their possible cytotoxicity. Molecular dynamics is a flexible and versatile computer simulation tool, which allows us to research the molecular level mechanisms of nanomaterials interaction with cell or bacterial membrane, predicting in silico their behavior and estimating physicochemical properties. In particular, based on the molecular dynamics simulations, a bio-action mechanism of two-dimensional aluminum hydroxide nanostructures, termed aloohene, was discovered by the research team led by Professor S. G. Psakhie, accounting for its anticancer and antimicrobial properties. Here we review three groups of nanomaterials (NMs) based on their structure: nanoparticles (globular, non-elongated), (quasi)one-dimensional NMs (nanotube, nanofiber, nanorod) and two-dimensional NMs (nanosheet, nanolayer, nanocoated substrate). Analysis of the available in silico studies, thus can enable us a better understanding of how the geometry and surface properties of NMs govern the mechanisms of their interaction with cell or bacterial membranes.
\end{abstract}

Keywords Nanoparticle $\cdot$ Nanotube $\cdot$ Nanosheet $\cdot$ Lipid membrane $\cdot$ Molecular dynamics

\footnotetext{
A. A. Tsukanov

Skolkovo Institute of Science and Technology, Moscow 121205, Russia
A. A. Tsukanov $(\bowtie) \cdot$ O. Vasiljeva
Institute of Strength Physics and Materials Science SB RAS, Tomsk 634055, Russia
e-mail: a.a.tsukanov@yandex.ru
O. Vasiljeva
e-mail: olga.vasiljeva@ijs.si
O. Vasiljeva
Jozef Stefan Institute, 1000 Ljubljana, Slovenia




\section{Introduction}

Over the past decade, the variety of different nanoparticles (NPs) and nanomaterials (NMs) were considered an important contributors to multiple medical diagnostics and the therapy applications [1, 2]. Nanomaterials can be used as devices [3], contrast drug carriers, drug delivery systems [4, 5], adjuvant, therapeutic and theranostic agents [6-8]. All these applications need an understanding of how the nanomaterial interacts with the membranes of the cell and intracellular organelles. Before the consideration of a NM-biomembrane interaction, it is important to describe what the typical cell membrane is.

Amphiphilic organic molecules, lipids, are the basic building units of the typical cell membrane [9]. Lipid consists of two parts: long hydrophobic tails and a comparatively compact hydrophilic head. Due to their composition, lipids form stable structures in water solution: e.g. liposomes, spherical and cylindrical micelles, bilayer membrane, etc. Lipid bilayer is stabilized by hydrophobic interaction between lipid tails in the inner part of the membrane, as well as by the interaction of head groups with water and with each other at the surface regions [10]. Lipid bilayer is a basis of the cell membrane.

The rapid development of multiple techniques, as well as growth and availability of high-performance computers, contribute to an increasing number of computer simulation studies of complex molecular systems, which may include sophisticated biological objects and complex nanomaterials. There are several computational approaches to study the NM-biomembrane interaction: a self-consistent field and density functional theory approach $[11,12]$, stochastic-elastic modeling [13, 14], all-atom [15] and coarse-grained [16] molecular dynamics, including classical unbiased and constrained or steered molecular dynamics (SMD) [17], Monte Carlo methods [18], etc.

It is worth mentioning several previously published reviews concerning the interaction of molecules and NMs with biomembrane models. Small compounds, drug molecules, biomolecules, and fullerenes interaction with the cell membranes was considered in [19, 20]. In particular, cases of fullerenes, their aggregates and derivatives impact to the membranes were summarized as, pure fullerenes $\mathrm{C}_{60}$ and their clusters tend to penetrate inside the lipid bilayer accumulating in the hydrophobic interior of the membrane (membrane width is several times larger than the diameter of $\mathrm{C}_{60}$ fullerene) [21-23]. It was noted that larger fullerenes or fullerenes in high concentrations can cause significant disturbances in the membrane structure [2426]. However, accordingly to [27] the presence of $\mathrm{C}_{60}-\mathrm{C}_{180}$ fullerenes inside the membrane with fullerene-to-lipid ratio about $\sim 1: 1$ results in a mechanical strengthening of the lipid bilayer. It was also reported, that the pure $\mathrm{C}_{60}, \mathrm{C}_{70}$ fullerenes can bind with ion channels, embedded into the lipid membrane, thereby affecting their structure or function [28, 29]. Fullerenes with a functionalized surface exhibit tendency to anchor their polar or charged groups either in a lipid-water interface $[21,23]$ or in hydrophilic-hydrophobic interface between lipid heads and tails parts $[26,30]$. The review of molecular dynamics studies of small compounds permeation 
through the lipid bilayer as well as the interaction of proteins with the membranes can be found in [31]. Simulations of the impact of different carbon NMs as fullerenes, nanotubes, and combustion-generated carbon NPs, having an arbitrary structure, on the cell membrane are reviewed in [32]. In-depth review describing the computer modeling studies of nanomaterials interaction with the cell membranes and other different biological nano-objects can be also found in a work [33].

\section{Nanoparticles}

In this section, we consider papers focused on the numerical simulations of the interaction of lipid membranes with organic and inorganic nanoparticles such as, dendrimers, functionalized gold NPs, bimetallic NPs of immiscible metals, Janus NPs and abstractive nanoparticles with a surface charge.

\subsection{Dendrimers and Dendritic Nanostructures}

Dendrimers and dendritic nanostructures attract much attention in biochemistry, nanotechnology, and pharmaceutical sciences, due to the possibility to precise control of its size, shape, and location of functional groups [34, 35]. Dendrimers play an important role in biomedicine, as contrast agents, gene-transfection agents, and antibacterial substrates [36]. The number of branches and the size of dendrimer of a certain kind depends on its generation number $\mathrm{G}$. A schematic of poly(amidoamine) dendrimers (PAMAM) G2 and a fragment of G11 dendrimers in the vicinity of the cell membrane is shown in Fig. 1. The 3D model of PAMAM G11 was built using the structural data from [37].

Lee and Larson using MD simulations showed that charged PAMAM, interacting with a cell, can induce pore formation in the lipid membrane. Herewith, this was observed at a temperature of $310 \mathrm{~K}$, while at lower $\mathrm{T}=277 \mathrm{~K}$ the described phenomenon did not occur, which is explained by transition of lipids into a condensed phase [38]. Moreover, it was also found that at a high ion concentration in water (about $0.5 \mathrm{M}$ of $\mathrm{NaCl}$ ) large charged dendrimers demonstrate no tendency to penetrate into the bilayer because of the screening of electrostatic interaction between dendrimer surface and lipid head groups. A simulation of PAMAM dendrimers and several copies of a peptide - poly-L-lysine (PLL) near the membrane showed that the ability to disturb the membrane is dependent on the generation number of the dendrimer, and hence on its size, as well as on the dendrimers concentrations near the membrane surface [39, 40]. Large dendrimers in a high concentration are capable to induce significant distortion of the bilayer structure, as well as the formation and stabilization of pores in the membrane. Kelly and co-workers performed MD simulations of neutral, positive, and negative dendrimers with the membrane in implicit water model [41]. It was shown that charged PAMAM dendrimers of third generation (G3) 


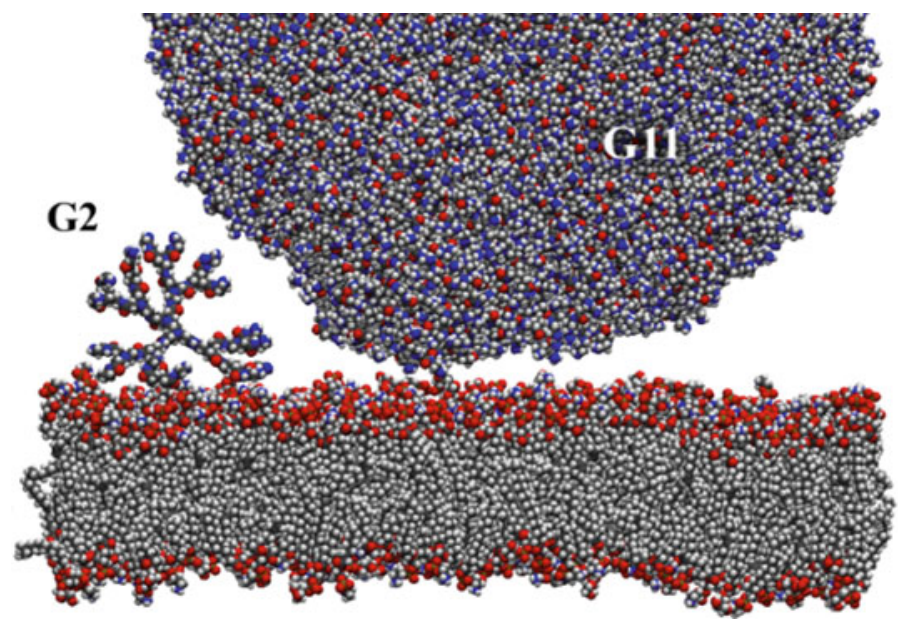

Fig. 1 Schematic of PAMAM dendrimers with generation numbers $\mathrm{G} 2$ and $\mathrm{G} 11$ near a lipid bilayer. Colors: carbon—grey, hydrogen—white, oxygen—red, nitrogen—blue, phosphorus—brown

more intensively interact with the membrane surface than neutral dendrimers. The interaction of charged dendrimers with the membrane in a liquid-phase state may also be accompanied by a hydrophobic interaction between the dendrimer interior and the lipid tail groups [42]. The permeation of the charged dendrimers through the lipid membrane under an elastic tension was studied using the coarse-grained MD simulations in [43]. The obtained results showed that the elastic tension enhances the permeability of the membrane for the charged PAMAM dendrimers of G3, G5 and G7 generation. Ting and Wang, using a self-consistent field theory, showed that in the absence of the lateral tension membrane prefer to wrap the charged dendrimer partially [44]. An increase in the dendrimer charge density increases the extent and stability of the wrapped state. But in a case of slightly tensioned membrane large charged nanoparticles as G5 (or higher) dendrimers can induce the formation of metastable pores in a lipid bilayer whereas for $\mathrm{G} 3$ dendrimers the pores are unstable.

\subsection{Abstractive Nanoparticles}

An abstractive NP means a model particle, which is not representing a certain material with a defined chemical composition, but mimics the NP with defined properties such as shape, size, charge, hydrophilic-hydrophobic balance etc.

In order to find out how the size and charge of nanoparticles affect their interaction with the cell membrane, Ginzburg and Balijepalli [45] used the self-consistent field/density functional theory of block copolymer/nanoparticle mixtures proposed in $[11,12]$. The results revealed that a neutral nanoparticle of a diameter $16-32$ A penetrates into the bilayer, forming a single-layer hybrid micelle in which lipids orient 


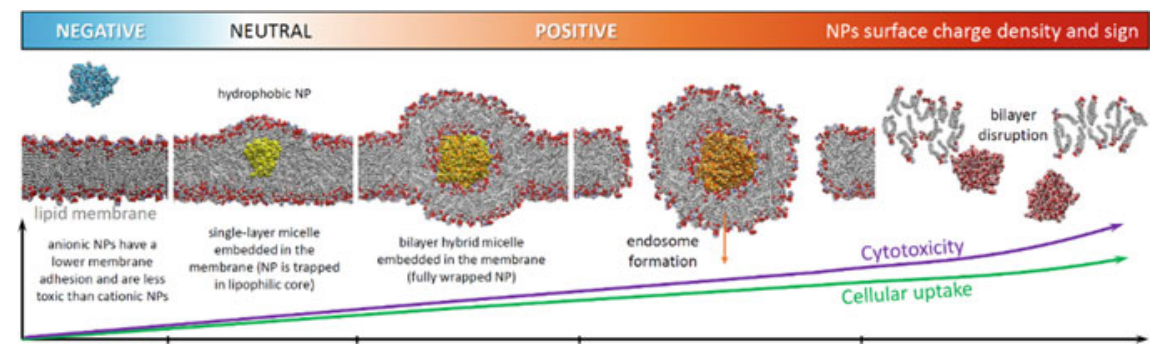

Fig. 2 A generalized scheme of the interaction of charged and neutral NPs with a cell membrane, combining the results from [45] and [49]. Anionic NP is less likely to translocate across the cell membrane than cationic NP. Hydrophobic NPs can integrate into the membrane, forming a singlelayer embedded micelle. Cationic NPs having high enough surface charge density intensively participate in endosomes formation and cellular uptake. Strongly charged cationic NPs may generate pores in the bilayer, causing the overt membrane disruption

their hydrophobic tails toward the nanoparticle (see Fig. 2). If the NP charge density increases, a hybrid bilayer micelle is formed in the membrane. Further increase in the charge density or/and size of NP causes separation of the endosome, containing the nanoparticle, which may be followed by the loss of membrane integrity (Fig. 2).

The interaction of hydrophobic and semihydrophilic NPs with the membranes was studied in [46]. Using the coarse-grained MD simulations it was shown that a hydrophobic nanoparticle with a radius of about $5 \mathrm{~nm}$ can easily penetrate into a lipophilic bilayer interior, while a semihydrophilic NPs remain on the lipid membrane surface. An amphiphilic nanoparticle with Janus structure, consisting of both a hydrophilic and a hydrophobic part, can exhibit more complex behavior. A coarsegrained MD study of the interaction of a Janus nanoparticles with a cell membrane were conducted by Alexeev et al. [47]. The considered Janus NPs have a size comparable to the bilayer thickness. The capability of such nanoparticles to stabilize pores in the lipid membrane was found. Stabilized nanopores can be opened at relatively low elastic stress applied to the membrane, thereby providing permeability of membranes to water, ions and other compounds. Thus, using Janus NPs, it is possible to control membrane permeability altering its tension, which may be caused by changing local environmental conditions such as temperature or $\mathrm{pH}$.

Besides the physicochemical properties of the NP surface, the shape and local curvature of the surface play an important role in NP interaction with a cell membrane. Yang and Ma investigated the translocation of NP with different shapes and sizes using coarse-grained model [48]. It was found that the shape anisotropy and the initial orientation of a particle are decisive for the character of its interaction with the lipid membrane. The penetrability of NP through a lipid bilayer is determined by the contact area between the particle and bilayer, and by the local surface curvature of the particle at the point of contact. The increase in curvature facilitates the translocation. It was also reported that NP volume indirectly affects penetration to a lesser extent.

The influence of the elastic lateral tension on the character of membrane interaction with abstractive dendrimer-like soft nanoparticles, having different generation 
number is investigated in [50]. It was found that the way, in which the membrane interacts with soft particles, depends on both the value and the sign of membrane surface tension. The researchers defined three typical phases of interaction of a soft NP and the membrane: penetration at high positive tension, penetration and partial wrapping at low positive tension, and full wrapping at low negative tension.

In addition, the interaction of multi-molecular complex comprising a membranesoluble outer shell and nanoporous core with the cell membrane can be considered in this subsection. Studying the membrane-NP interaction, Carr et al. proposed a possible route for forming a synthetic ion channel in the cell membrane by embedding a supramolecular complex of dimethyldioctadecylammonium (DODA) surfactant capsule with a porous polyoxomolybdate (POM) particle into the lipid bilayer [51]. The POM nanoparticle has a strong negative charge $(-72 \mathrm{e})$ that makes their embedment in the membrane impossible (Fig. 2). Carr and colleagues using coarsegrained MD showed that the mixed capsule of amphiphilic cations DODA and POPC lipids, formed around the POM nanoparticle, facilitates the NP embedment into the membrane. The positive charge of the detergent liposomal structure partially screens the negative POM charge, and the further liposome fusion with the lipid bilayer leads to the embedding and stabilization of NP in the membrane center.

\subsection{Metallic Nanoparticles}

Gold nanoparticles (AuNP) have a wide range of possible biomedical applications [52] that explains the great attention paid to studies of the interaction of AuNP with the membrane, including in silico approaches. The interaction of functionalized gold nanoparticles with electroneutral and negatively charged lipid membranes was investigated in [49]. The considered AuNPs were functionalized with charged and/or hydrophobic ligands. Cationic ammonium groups and anionic carboxylate groups were used to provide AuNP with a positive or negative surface charge, respectively. Different ratios of charged/hydrophobic ligands coated gold core to make different surface charge densities of the NPs. The results of coarse-grained computer simulations showed that the AuNP may spontaneously adhere to the membrane surface or penetrate into the bilayer. The way they interact with membrane depends on both sign and density of NPs surface charge (Fig. 2). Using SMD simulations it was found that the approach of anionic AuNP to the negatively charged membrane is complicated by electrostatic repulsion. In both cases of the neutral and negative membranes, anionic NPs have free energy minima near bilayer surface in the adsorbed state. The free energy profile for gold NPs with hydrophobic ligands only reach minimal values inside a lipophilic bilayer region. In case of neutral lipid membrane, the energetically more preferable configuration for cationic AuNP (with 70\% of charged ligands) as well as for anionic one is the adsorbed state without any significant bilayer distortions. Interaction of AuNP, having cationic ligands, with the negatively charged membrane is more intensive, strong binding, and immersion of NP were observed, which cause a large deformation of bilayer as well as a formation of hydrated region within the 
membrane. The free energy profile has a minimum value corresponding to NP position inside the lipid head groups region. Increasing the surface charge density of positively charged AuNP enhances the ability of such particles for cellular uptake; however, beginning with a certain surface charge, severe deformation and disintegration of the cell membrane take place (Fig. 2). It was pointed out that there is a range of surface charges of cationic NPs in which a balance between cellular uptake and cytotoxicity may be attained [49].

The influence of the shape and surface functionalization of gold nanoparticles on the character of its interaction with a negatively charged membrane was investigated in [53]. Based on the coarse-grained simulations, the estimates of the free energy barriers and translocation rate constants were obtained depending on the nanoparticle shape and charge density. It was shown that anionic NPs were electrostatically repelled from the membrane surface and their translocation through the bilayer is less probable in comparison with cationic NPs. Furthermore, shape anisotropy may result in the reorientation rotations of the charged NPs in the contact region with membrane, thus distorting the lipids self-assembly and possibly causing cytotoxic effect. For the studied cases, it was also found that translocation rate constants may differ 60 orders of magnitude, in spite of equal sizes of NPs [53].

It is important that both the sign and the density of NP surface charge can be tuned by grafting of the ligands with certain charge, polarity and hydrophilic-hydrophobic balance. Recent theoretical research of Professor S. G. Psakhie's scientific group, devoted to bimetallic nanoparticles of immiscible or partially miscible metals and their interaction with bacterial and cell membranes, showed that the electrostatic properties of NP surface can be tuned without grafting of the charged ligands [54]. Using the embedded atom method (EAM) $[55,56]$ and MD simulations it was shown that the surface of bimetallic Ag-Cu NP is formed by silver atoms independent on nanoparticle composition $\left(\mathrm{Ag}_{70} \mathrm{Cu}_{30}\right.$ or $\left.\mathrm{Cu}_{70} \mathrm{Ag}_{30}\right)$ due to lower surface energy of $\mathrm{Ag}$ (Fig. 3a). Furthermore, due to different electronegativity of $\mathrm{Ag}$ and $\mathrm{Cu}$ atoms, silver and copper, in average, will have different partial charges. Using the density functional theory (DFT) [57, 58], the mean values of partial atomic charges of Ag and $\mathrm{Cu}$ metals in small $\mathrm{Ag}-\mathrm{Cu}$ clusters were estimated as a function of $\mathrm{Ag}$-to-Cu ratio in the nanocluster. Based on this result it was concluded that magnitude of the surface charge density of considered bimetallic $\mathrm{Ag}-\mathrm{Cu} \mathrm{NP}$ is adjustable in the range from -2.9 to $-7.3 \mathrm{e} / \mathrm{nm}^{2}$, by the changing of $\mathrm{Ag}$-to-Cu ratio from $7 / 3$ to $3 / 7$. Moreover, choosing metals by taking into account both the difference in their electronegativity and the difference in their surface energies, it is possible to synthesize bimetallic NP having a given permanent surface charge density of a certain sign [54]. In addition, using the force field based SMD simulations, in which metal atoms are uncharged, the free energy of interaction of $\mathrm{Ag}_{70} \mathrm{Cu}_{30}, \mathrm{Cu}_{70} \mathrm{Ag}_{30}$ and $\mathrm{Cu}_{70} \mathrm{Ag}_{30} \mathrm{O}_{4}$ nanoparticles of a diameter about $40 \AA$ with bacterial and cell membranes were estimated. It was found, that lipopolysaccharide (LPS-DPPE) membrane adsorbs the bimetallic NPs on the membrane-water interface (Fig. 3b), whereas lipid bilayer membrane demonstrates the tendency to wrap (or partially wrap) pure $\mathrm{Ag}_{70} \mathrm{Cu}_{30}, \mathrm{Cu}_{70} \mathrm{Ag}_{30}$ NPs (Fig. 3c). In the case of $\mathrm{Cu}_{70} \mathrm{Ag}_{30} \mathrm{O}_{4} \mathrm{NP}$, the presence of surface oxide groups prevent the wrapping of the NP by the lipid membrane. 

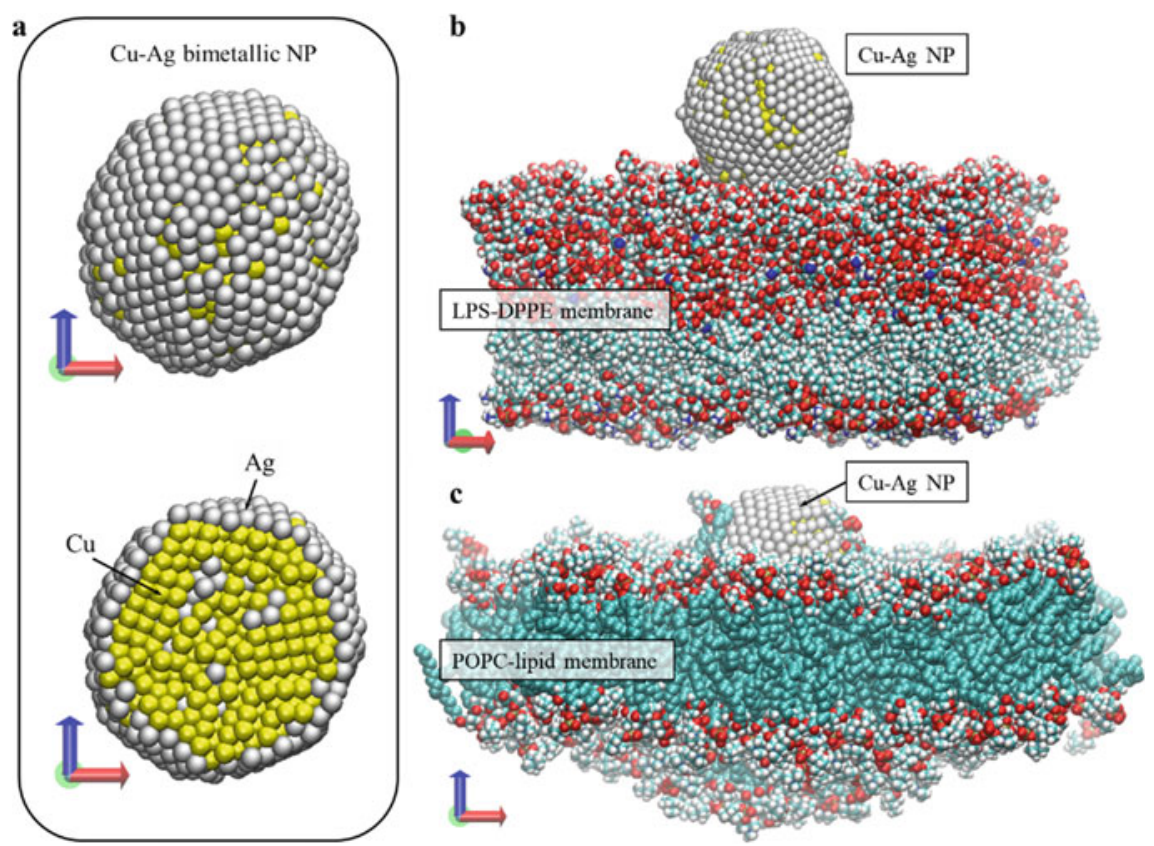

Fig. 3 Bimetallic NPs of immiscible or partially miscible metals: a structure of $\mathrm{Cu}_{70} \mathrm{Ag}_{30} \mathrm{NP}$, silver as a metal with lower surface energy forms a surface of $\mathrm{NP} ; \mathbf{b}$, $\mathbf{c}$ interaction of $\mathrm{Cu}-\mathrm{Ag} \mathrm{NP}$ with lipopolysaccharide (LPS-DPPE) membrane and POPC-bilayer, respectively. The images were available using the 3D data from work [54]. Colors: Ag-grey, $\mathrm{Cu}-$ yellow, $\mathrm{C}-$ cyan, $\mathrm{O}-\mathrm{red}$, $\mathrm{H}$-white, $\mathrm{N}$-blue (water is not shown)

\section{One-Dimensional Nanomaterials}

\subsection{Carbon Nanotubes}

Nanomaterials with a structure as nanotube (NT), nanofiber, nanowire, nanorod, etc. belong to the class of (quasi)one-dimensional NMs (1D-NMs). The carbon nanotubes (CNT) occupies a special place among 1D-NMs and remains for a long time in the focus of researchers attention. CNTs are promising structures for the biotechnology and biomedicine, due to their biocompatibility, high stability, mechanical elasticity, thermal, electrical, and optic properties as well as the adaptability for chemical modification with bioactive compounds [59]. The single-walled carbon nanotubes (SWNTs) structure can be open or can be terminated with caps (hemispheres of a fullerene). In the former case, NT has a nanocapsule-like structure with the internal cavity isolated from the environment. Ligands in functionalized CNTs can be chemically bound with the cylindrical surface or ends. Functional groups can also be bound with CNT by non-covalent bonds. Nanotubes can be wrapped with surfactants, proteins, lipids, DNA, etc., forming the supramolecular complexes 
due to the Van der Waals forces, hydrophobic interaction, and electrostatic polarization. Depending on chirality, SWNTs can exhibit metallic or semiconductor properties. Electronic properties of the SWNTs provide a formation of stable DNA-CNT nanohybrids $[60,61]$. It is important to note, that typically in the force field-based MD simulation the polarizability of CNT is not taken into account, and carbon atoms are modeled as Lennard-Jones particles with zero partial charges.

One of the pioneering MD studies of the interaction of CNT with the membrane is reported in [62]. The results showed that open carbon SWNT of $13 \AA$ in a diameter with hydrophilic groups at the ends, due to the formation of salt bridges with lipid head groups, is capable of the spontaneous embedment into the membrane, forming a transmembrane channel permeable for water molecules. Two phases of a transmembrane channel formation were distinguished: (1) location and embedment of the NT into the membrane surface, and (2) turn to a transmembrane position. As a hydrophilic NT end moved through the membrane, lipids attached from the nearest membrane monolayer were transferred to the opposite one. The example of a transmembrane position of a zigzag-type SWNT with chirality indices $(25,0)$ and with ends functionalized by OH-groups is shown schematically in Fig. 4 (left CNT).

The formation and transport properties of CNT-based transmembrane channel were investigated by Zimmerli and Koumoutsakos, using the all-atom MD simulations [63]. The results showed a possibility of electrophoretic transport of short RNA segments (20 adenosine nucleotides) through a synthetic channel based on transmembrane SWNT with a diameter of $18.7 \AA$. It was found that an electrostatic potential difference of about 1-2 V maintains the RNA fragment translocation with a velocity of about 1-30 nucleotides per nanosecond.

The study of interaction of non-functionalized NTs, having different length, with the membrane showed that short NTs, being inside the lipid bilayer, prefer to be oriented parallel to lipid molecules [64]. An increase in the length of NT change the preferred orientation to parallel to the membrane plane. The example of such

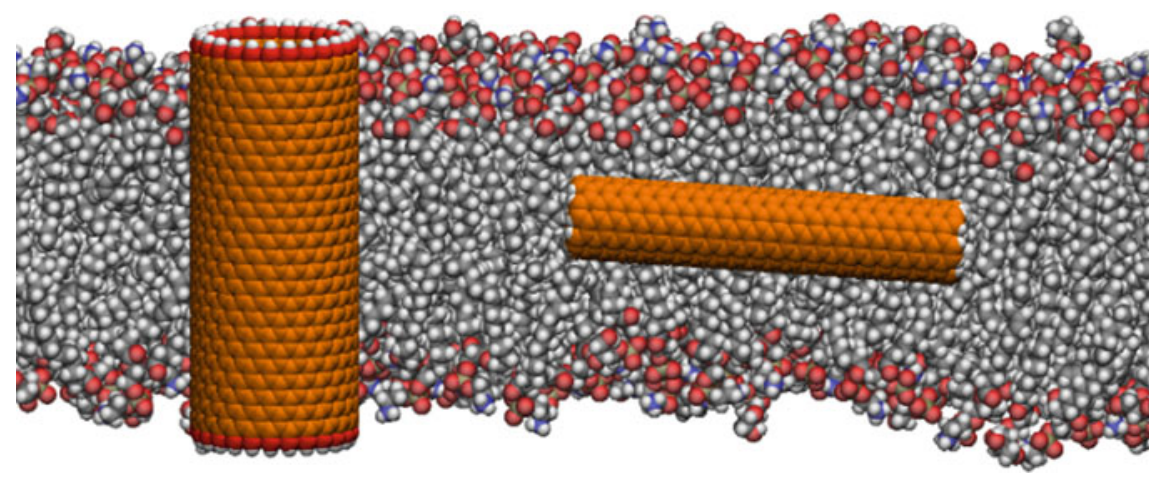

Fig. 4 Schematic of a transmembrane SWNT with functionalized faces (at the left) and hydrophobic thin CNT inside the bilayer. Color legend: carbon of CNT_-orange, C (lipids)—grey, H-white, $\mathrm{O}-\mathrm{red}, \mathrm{N}$-blue 
an orientation of armchair-type SWNT with chirality $(6,6)$ in the bilayer center is shown schematically in Fig. 4 (right CNT).

Based on a coarse-grained MD model, two ways of CNT penetration through the membrane were revealed: by wrapping with lipids and by direct piercing [65]. It is reported that the key factor, which defines the way of penetration, is the diameter of NT. Nanotubes of a rather small diameter pierce membranes, whereas those of larger diameter penetrate through the bilayer via wrapping. The wrapping of a thinner NT is accompanied by considerable bending of the membrane, which is less energetically favorable than the separation of lipids to a comparatively small distance. For a NT of diameter comparable with the membrane thickness, the distance to which lipids need to be displaced for piercing is significant, however, the membrane curvature would be low if membrane wraps the NT. This explains why penetration via wrapping become more energetically preferable than piercing.

According to the computation results [66] based on a single chain mean field theory (SCMF) [67], the energy required for penetration of a perpendicularly oriented NT with a hydrophilic surface is about hundred of $k_{B} T$. It was noted, that an orthogonal penetration produces the least damages to the membrane and has the least energy barrier. A nanotube with a hydrophobic surface is "attracted" by the hydrophobic core of the bilayer, which prevents its movement and separation from the membrane due to thermal motion. The SCMF theory was also applied for estimating the penetration parameters of a NT decorated with alternate hydrophilic and hydrophobic bands on its surface [68]. It was shown that a specific pattern on the surface of a NT can facilitate its penetration through a lipid bilayer.

The penetration of NTs through the membrane is dependent on a composition of the membrane, e.g. on cholesterol content, as it was shown in a comparative study of a POPC-lipids bilayer and POPC/cholesterol membrane [69]. Based on a constant velocity SMD simulation of NT penetration, it was demonstrated that the presence of $30 \%$ of cholesterol molecules stabilizes the membrane and increases its rigidity. Moreover, MD modeling of lipid membranes reinforced with SWNTs showed that intercalated nanotubes limit the degrees of freedom of neighboring lipids, strengthen membranes, and make them more stable and resistant to temperature increase [70]. The decrease in lipid mobility, particularly, the diminution of selfdiffusion coefficient of lipids in the presence of NTs intercalated in the bilayer was also reported [71].

Kraszewski and co-workers, investigated the interaction of pristine (nonfunctionalized) and amino derivative-functionalized SWNTs with the membrane [72]. The results of MD simulations showed that a closed pristine SWNT freely penetrates into the bilayer through three phases of passive diffusion: landing and floating on the bilayer surface $(60 \mathrm{~ns})$, fast penetration through the zone of head groups (20-40 ns), and finally, sliding through the lipid tails region. The penetration of functionalized SWNTs is similar, except that with a comparatively large number of functional groups where the phase of landing is in fact absent. During the functionalized SWNT translocation through the bilayer, amine groups were deprotonated in the simulation and then, when NT approached the opposite membrane side, the charges were recovered. It is pointed out that the presence of functional groups slows 
down the penetration of NT through the bilayer. Open SWNT can cause sticking of lipids at their open ends, thereby violating the local structure of the membrane. This effect increases the free energy barrier for translocation of open SWNTs through the membrane in comparison with capped ones. The simulation results [73] lead to the same conclusions. The penetration of open and closed SWNTs of the armchair type through a cholesterol-containing lipid membrane was studied using the all-atom model. It was shown that the penetration of a closed NT causes smaller membrane disturbances than an open one does. A closed SWNT has a lower free energy barrier to penetration through the membrane, which makes it a successful choice for the drug delivery, serving as nano-carriers or nano-containers.

As an example of the use CNT as nano-carrier, the delivery of paclitaxelum (PTX) encapsulated into an open nanotube (PTX@SWNT) through a lipid membrane was considered in [74]. Paclitaxelum is a mitotic inhibitor used in cancer chemotherapy, which molecule has a polar core. Four SMD calculations were performed for the penetration of a PTX@SWNT complex through a lipid bilayer at different velocities. The PTX molecule was located in the far region of the SWNT. In the simulation, external forces were applied both to the PTX and to the SWNT. The highest resistance to penetration was observed when the complex passed through the region of lipid tails which was due to the formation of hydrophobic bonds between the lipophilic groups and the outer SWNT surface. Entry of both the water molecules and the lipids into the inner volume of the nanotube was observed during the simulation. Moreover, the simulations revealed the formation of hydrogen bonds between the PTX and water molecules penetrated into the SWNT. It was noted that a stabilization of the PTX molecule inside the SWNT occurs due to both the Van der Waals forces between the PTX and inner surface of SWNT wall, and the hydrogen bonding between the PTX and water molecules penetrated inside the nanotube cavity.

In order to investigate the features of cell membrane interaction with pristine and functionalized SWNTs differing in diameter, length, chemical modification, and location of functional groups, the simulations with seven types of SWNTs with closed ends and aggregates of SWNTs have been conducted in [75]. The nanotubes had different positions of hydrophilic groups: fully hydrophilic SWNTs, hydrophilic end groups, and fully hydrophobic SWNTs. It was shown that small nanotubes spontaneously penetrate into the membrane. The most stable position of closed hydrophobic SWNTs is the membrane center with an orientation parallel to the bilayer plane. However, in the case of NT with a length smaller than the membrane thickness, the energy minimum for perpendicular (or transmembrane) orientation in the bilayer is somewhat lower. For SWCNTs with functionalized ends and a length slightly greater than the membrane thickness, it is advantageous to have a transmembrane position with some angle to the bilayer at which the difference between the nanotube length and the membrane thickness is compensated. Fully functionalized hydrophilic SWNTs are preferably adsorbed by the membrane at the water-lipid interface in an orientation parallel to the membrane plane. The probability of crossing the membrane or embedding in the bilayer for such SWNTs is extremely low. It is also shown that bundles of several nanotubes self-aggregate in a water solution. These aggregates 
entering the bilayer significantly disrupt the membrane structure and the perturbation increases with increasing cluster size.

\subsection{Boron Nitride Nanotubes}

Despite the similar to CNT tubular geometry, analogous crystal structure, and unique physicochemical properties, the nanoscale mechanisms of the boron nitride nanotubes (BNNT) interaction with the biomembranes and other bio-object are mostly unknown and very poorly covered by MD-based studies. To date, there are only two MD studies of BNNT-lipid bilayer interaction with the atomic description of the system [76, 77].

The pioneering MD study of the BNNT interaction with the lipid membrane was conducted by Hilder and co-workers [76]. The BNNTs with chirality $(10,0)$ and $(10$, 10), having a length of $2 \mathrm{~nm}$, were considered. The partial atomic charges of $\mathrm{B}$ and $\mathrm{N}$ atoms were $0.4 \mathrm{e}$ and $-0.4 \mathrm{e}$, respectively. It was shown that the mechanism of BNNT insertion is similar to the mechanism of insertion of amino-functionalized carbon nanotubes, previously considered by Kraszewski et al. [72], into the lipid bilayer, except for the final stage of realignment. The following four stages were determined for the BNNT insertion into the membrane: (1) formation of BNNT-bilayer contact, (2) BNNT reorientation to become almost parallel to the membrane plane, (3) an interaction of one end of BNNT with the lipid head groups, and (4) an insertion of BNNT, allowing it to slide into the bilayer at an angle of about $45^{\circ}$. As a result the BNNT is partially inserted and is stable inside the bilayer. In general, the behavior of BNNTs is similar to the behavior of functionalized carbon nanotubes, which is less cytotoxic than pure CNTs. Although Hilder et al. performed both the unbiased and steered MD simulations, the energy characteristics of BNNT-membrane interaction were not obtained.

In order to estimate the free energy profile, including the energy barrier, and the depth of energy well, the SMD simulations with a potential of mean force (PMF) analysis of short BNNT and analogous CNT insertion into the lipid bilayer were conducted by the Professor S. G. Psakhie scientific group [77]. Previously, using DFT calculation, it has been found that the partial atomic charges of $\mathrm{B}$ and $\mathrm{N}$ are strong environmentally dependent [78]. In particular, in case of BNNT with chirality $(5,5)$ in a vacuum, boron atoms have partial charge of $+0.4 \mathrm{e}$, nitrogen $-0.4 \mathrm{e}$, but for the BNNT containing 5-7 water molecules inside, the absolute values of partial charges are significantly higher $\pm 1.05 \mathrm{e}$. For this reason, two models of BNNT were examined in the study [77]: BNNT $( \pm 0.4)$ and BNNT $( \pm 1.05)$. A diameter of BNNT was $6.9 \AA$, length $\sim 11.3 \AA$.

The result of SMD simulations with the PMF analysis showed that: (1) both BNNT have local minimum of energy about $-10 \mathrm{~kJ} / \mathrm{mol}$ at the lipid-water interface, (2) the BNNT $( \pm 0.4)$ has a global minimum of $-72 \mathrm{~kJ} / \mathrm{mol}$ inside the lipophilic core of the bilayer, the depth is about $30 \mathrm{~kJ} / \mathrm{mol}$ smaller than that in case of analogous CNT, (3) the CNT was staying empty inside the lipid membrane whereas BNNT 


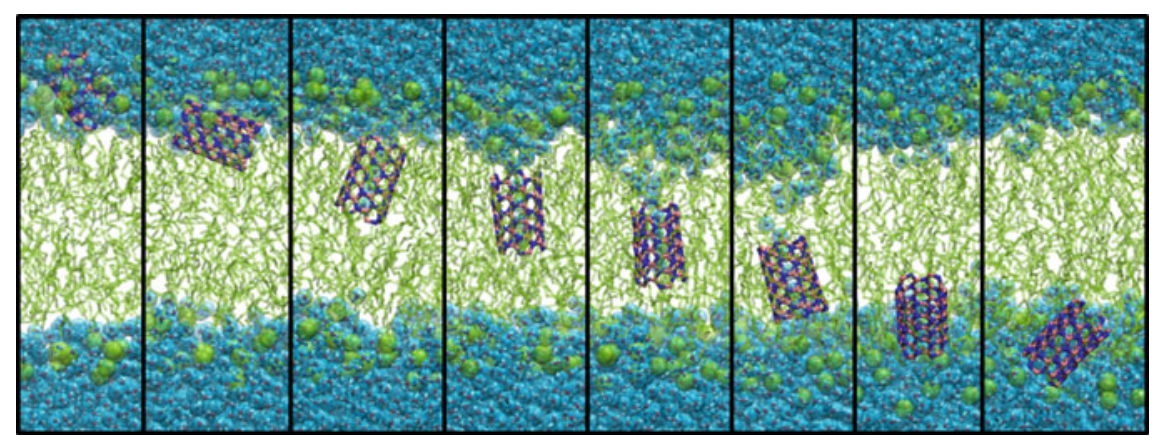

Fig. 5 Boron nitride nanotube (BNNT) translocation through the POPC membrane in steered MD simulation. The orientation of BNNT is transmembrane when it is crossing the tail region of the membrane. BNNT tilts orientation when overcoming the head group-water interface. Water is observed inside BNNT. Images use the XYZ-data from work [77]. Colors: B—pink, N—blue, lipids-light-green, water-light-blue

brings in several water molecules, (4) the penetration of the BNNT into the bilayer is accompanied by a formation of "water defect"-a several-water-molecule long tail behind the BNNT (Fig. 5), (5) the difference in orientation of short BNNT and CNT translocating into the bilayer is that the CNT tends to be in a perpendicular orientation to the membrane plane during whole time of insertion, whereas BNNT $( \pm 0.4)$ has a tilt from 0 (parallel) to $45^{\circ}$ being in the head-group region (Fig. 5), (6) the insertion of the BNNT $( \pm 1.05)$ is not energetically favorable. The former means an absence of impact on the cell membrane, which indirectly indicates a less cytotoxicity of this nanomaterial.

Besides the two abovementioned researches there is a study of BNNT-membrane interaction, in which, using the mixed model - the Lennard-Jones potential together with the continuum approximation, the relationship between the location of energy minimum, the radius of a cylindrical hole in the membrane, and the perpendicular distance of the BNNT from the hole was determined [79].

\section{Two-Dimensional Nanomaterials}

\subsection{Graphene}

One of the pioneering MD studies on the interaction of graphene nanosheet (GNS) with a cell membrane was reported in [80]. Using a coarse-grained MD model, it was shown that GNS can form composite sandwich-like structures in the internal hydrophobic region of a lipid bilayer. Such hybrid graphene-membrane structure can be obtained by forming hydrated micelles of individual GNS coated with a phospholipid layer which can then be absorbed by the membrane. The GNS dimensions 
in the simulation were about $60 \times 60 \AA^{2}$. It was found that the absorbed graphene nanosheet residing at the bilayer center parallel to the membrane plane is a stable system. Slow diffusion motion of the frustrated graphene along the membrane was observed. It is pointed out that the nanosheet does not change the bilayer thickness. The absorption of a lipid micelle containing the GNS by the membrane was also investigated. It was shown that during the absorption of the micelle, the graphene sheet is fixed in the hydrophobic core of the bilayer and lipids from the shell transfer into the nearest membrane leaflet. Full absorption and stabilization of the nanosheet in the bilayer center required about 500 ns. The dependence of displacement of graphene balanced inside the bilayer on the force applied to its edge perpendicular to the system plane was also estimated. Further integration of the obtained data gave an estimate of the energy required for the extraction of graphene from the membrane. In the case of three parallel nanosheets are absorbed, the membrane increases in thickness by about $1 \mathrm{~nm}$, the absorption of eight-GNS sandwich leads to an increase of thickness more than 1.5 times. If the size of a nanosheet on one of the sides coincides with the bilayer thickness and its opposite faces are functionalized by hydrophilic groups, the graphene fragment takes a stable transmembrane position inside the bilayer perpendicular the membrane plane.

The penetration of single square and circular fragments of pristine graphene GNSes of different sizes into the membrane was studied in [81]. It was shown that as the nanosheet size is increased, the bilayer tends to form semi-spherical vesicle, and thus the membrane experiences substantial deformation. During internalization, the angle between the membrane plane and GNS was estimated. The behavior of this angle revealed three stages: (1) spontaneous orientation before the nanosheet touches the membrane surface, (2) embedment of the nanosheet into the bilayer mainly at an angle of $47^{\circ}$, and (3) rotation of the nanosheet into membrane-parallel position with the formation a sandwich-like superstructure. The MD simulation was also performed to study how the thickness of single- and multilayered graphene nanosheets and the degree of their surface oxidation influence the interaction with a lipid membrane [82]. The penetration of a GNS coated with lipids was also considered. It was shown that for the pristine GNS, the position in the bilayer center parallel to the membrane plane is stable, whereas a nanosheet with oxide at the boundary ( $10 \%$ degree of oxidation) is attached at an angle to the membrane plane. The degree of boundary oxidation strongly affects the final GNS orientation in the bilayer. At oxidation values of less than 5\%, the GNS behaves as a pristine one. It was noted that there are two appreciable energy drops in the system during penetration: (1) when the GNS is embedded in the bilayer, and (2) when it rotates inside the bilayer to take its final position. The second energy drop is lower than the first one. The simulation of coated GNS was performed for different amounts of coating lipids. At a low surface absorption density, the nanosheet edge zones were open to water. The penetration was no different from the internalization of pristine graphene sheets. At a relatively high lipid concentration on the GNS surface, no penetration within the rather limited time of MD simulation was observed. For penetration of a multilayered graphene into the bilayer, piercing of the membrane by its corner and parallel orientation inside the membrane, like for single GNS, was detected. 
Using coarse-grained MD and all-atom SMD simulations, the mechanism of spontaneous penetration of multilayered (few-layer) GNS into a cell though piercing the membrane by their edge or corner was studied in [83]. The model took into account the roughness (asperities) of the GNS edge the texture of which was borrowed from an image of the real structure taken with a transmission electron microscope. The results of MD calculations showed that monolayer graphene in the form of a rhomb (with an edge length of $64 \AA$ and most acute angle of $30^{\circ}$ and $60^{\circ}$ ) that turns due to thermal motion resulting in one of its acute corner sites to be directed to the bilayer. When the region of head groups is pierced, complete GN absorption by the membrane can be initiated through hydrophobic interaction of graphene with lipid tail groups. Additionally, a series of calculations with triangular, square, and hexahedral fragments of both pristine GNS and GNS functionalized at the corners or along the perimeter were performed. The results of MD calculations showed that absorption occurs with the non-functionalized corner forward, and if no such corners are present, the GNS remains on the membrane surface. Using the SMD method, the free energy barrier for graphene penetration into the membrane was estimated. It was shown that the penetration of the GNS with an ideal boundary into the membrane is almost impossible at room temperature, whereas inhomogeneous topography of its boundary can greatly decrease the free energy barrier. It was noted that when absorbing the GNS, the membrane structure is hardly disturbed, except that the lipid tail groups are straitened along the nanosheet surface due to high adhesion.

A series of coarse-grained calculations were performed to study the interaction of pristine graphene and graphene oxide with a lipid membrane for different nanosheet sizes and degrees of oxidation [84]. The graphene sheets were square flakes with side lengths of 35,70 , and $105 \AA$; the density of oxide groups was varied from 0 to $40 \%$ of the total number of carbon atoms. Nanosheets oxidized only along the perimeter were also considered. In total, four variants were simulated: pristine GNS, graphene oxidized along the perimeter (epitaxial graphene oxide, EGO), graphene with oxide along the perimeter and $20 \%$ on the surface (GO20), and graphene with oxide along the perimeter and $40 \%$ on the surface (GO40). Each calculation took about $45 \mu \mathrm{s}$. It was found that the graphene interaction with the membrane provides one of the four configurations: a sandwich-like structure with a nanosheets located in the bilayer center and parallel to it (for GNS with sides $l=35,70 \AA$ ); a graphene-containing semi-spherical vesicle immersed into the bilayer (for GNS and EGO with $l=105$ $\AA$ ); a nanosheet lying on the membrane surface and parallel to it (GO20 and GO40 with $l=35 \AA$ ); and crosswise oriented graphene inside the bilayer. It was found that the scale of observed membrane distortions depends on the degree of graphene oxidation: the more this degree, the higher the distortion of the bilayer structure. Increasing the side of square graphene fragments increases these disturbances up to the point of membrane continuity disruption.

The results of experimental and numerical studies of the interaction of pristine graphene and graphene oxide with a bacterial membrane were reported in [85]. According to them, GNS can embed and cut the external and internal cell membranes of $E$. coli. This involves degradation of the membrane with possible destruction of the cell wall and partial cytoplasm loss. Using the all-atom MD model, it was shown 
that there exist three phases of graphene and graphene oxide interaction with lipid membranes. The first phase is diffusion motion of the nanosheet till it touches the membrane; the second phase is its comparatively fast insertion into the bilayer; the third one is an extraction of lipids from the bilayer due to Van der Waals forces and hydrophobic interaction on the GNS surface. This involves substantial depression and deformation of the membrane, facilitating disruption of its continuity. Such an action decreases the bacterial cell viability and allows consideration of graphene as an antibacterial nanomaterial. Schematic molecular model illustrating the extraction of the lipids on GNS surface is presented at Fig. 6.
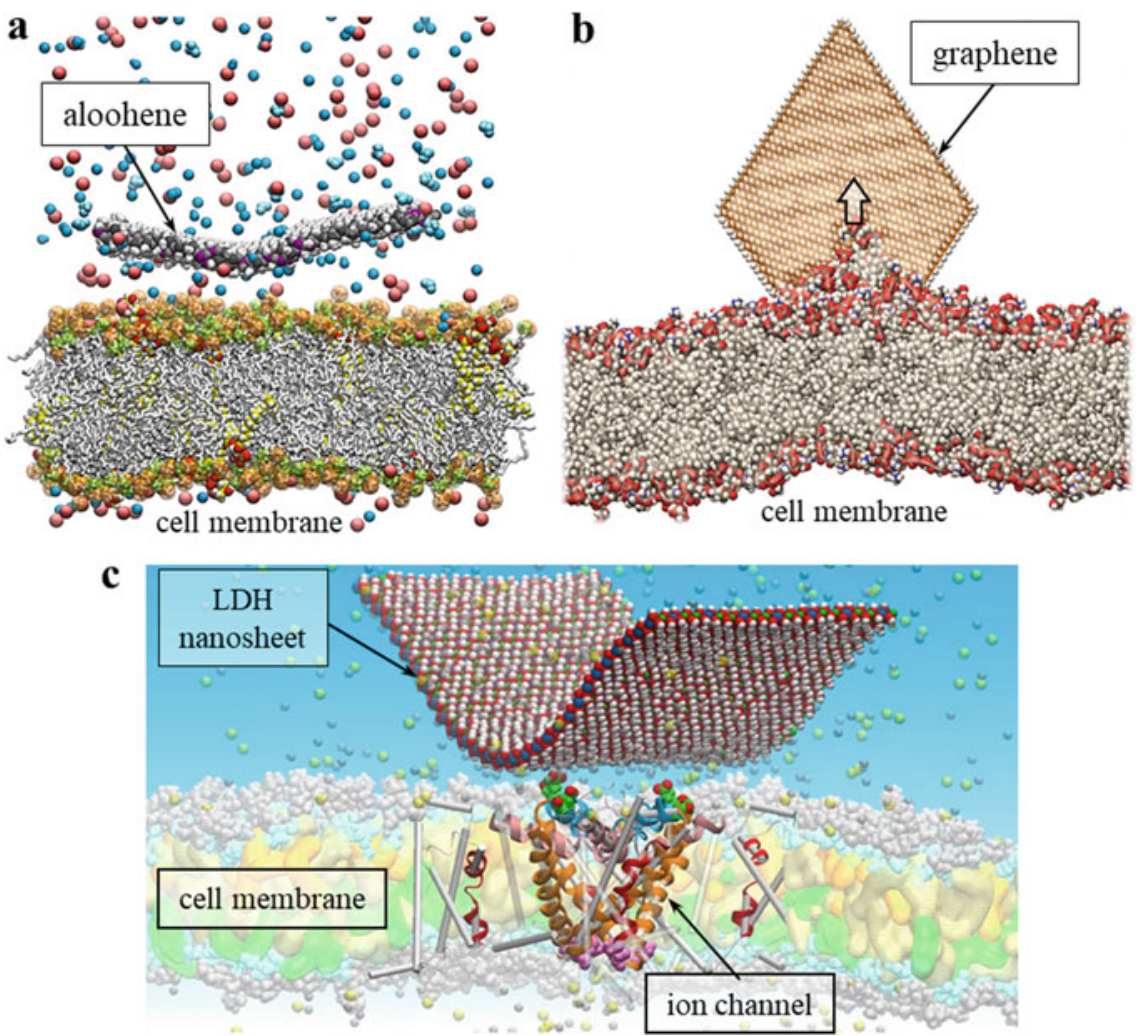

Fig. 6 Two-dimensional nanomaterials and cell membrane interaction: a aloohene doesn't penetrate to the bilayer and doesn't extract lipids, its impact to a cell is a dysregulation of ionic balance (the result of long unbiased molecular dynamics simulation). Reproduced with permission from ACS [6]. b Schematic molecular model illustrating the extraction of the lipids onto GNS surface (orange) from the cell membrane. $\mathbf{c}$ Nanosheet of layered double hydroxide interacting with outer loops of bacterial sodium channel $\mathrm{Nav} \mathrm{Ab}$, embedded into the membrane (the model was built using XYZ-data from [86]) 


\subsection{Metal (Oxy)hydroxides}

Unlike GNS, the layered metal hydroxides and oxyhydroxides are hydrophilic compounds with large amount of polar $\mathrm{OH}$-groups on the surface. Layered metal hydroxides and oxyhydroxides as a rule are chemically inert, non-toxic, biocompatible, have a large specific surface area with non-zero surface charge, can form the hybrid complexes with organic molecules, and be used as selective ion exchangers with a high proton and/or hydroxyl buffer capacity. These unique physicochemical properties make the layered metal (oxy)hydroxides an important material for a wide range of applications in different areas, including biomedicine.

One of the first MD research of biomembrane interaction with metal (oxy)hydroxide was performed by Shroll and Straatsma [87]. They considered an adhesion of the gram-negative bacteria outer membrane on $\mathrm{FeOOH}$ (goethite). Although the goethite $\mathrm{FeOOH}$ is not a layered oxyhydroxide but the oxyhydroxide with bulk crystal structure, we will consider this case, since the modeled fragment has a thickness of several atomic layers. The modelled outer membrane of Pseudomonas aeruginosa bacteria has bilayer structure and consist of lipopolysaccharide (LPS) monolayer (outer leaflet) and the monolayer of typical phospholipids (inner leaflet). The LPS has several carboxyl and phosphate groups, which are the acceptors of proton. Each LPS molecule brings strong negative charge of $-13 \mathrm{e}$. The strong adhesion within LPS-NM interface was observed. It was found that outermost LPS groups must compete with water molecules resided near the $\mathrm{FeOOH}$ surface and may replace them forming multiple hydrogen bonds with hydroxyl groups of the mineral surface. Due to the formation of these bonds the significant changes in the structure of outermost saccharide groups of the membrane were observed. No obvious tendency of $\mathrm{FeOOH}$-induced membrane disruption is reported.

A similar result was obtained in the unbiased MD simulation of the layered double hydroxide (LDH) near the outer surface of the membrane with bacterial ion channel embedded [87]. The hydrogen bonding of outer carboxyl-contained groups of the $\mathrm{Na}_{\mathrm{V}} \mathrm{Ab}$ voltage-gated sodium-selective ion channel with the hydroxide groups on the LDH surface was observed, without any tendency to disrupt the membrane (Fig. 6).

The layered metal oxyhydroxides nanomaterial based on the $\mathrm{AlOOH}$ (boehmite), termed "aloohene", was developed by the scientific group of Professor S. G. Psakhie [6] and showed promise for multiple biomedical applications. The all-atom MD simulations of the aloohene fragment in the vicinity of the cell membrane demonstrate that aloohene doesn't tend to internalize into the lipid bilayer. Moreover, due to the electrostatic and amphoteric properties of its surface, the aloohene dysregulates the composition and the ion balance of the tumor cell microenvironment.

It is supposed that the electrostatic action on the membrane and perturbation of the ion concentration near its surface can greatly affect the functioning of membrane proteins sensitive to changes in membrane polarization. This effect can be critical for some metabolic and intracellular processes, including those which support viability and proliferation of tumor cells. 


\section{Summary}

Computer simulations of interactions on the molecular level provide a unique opportunity to explore the processes and mechanisms in such complex and heterogeneous systems as biomembrane-nanomaterial interfaces at picosecond-microsecond timescales. The understanding of the basic rules, which govern adsorption, insertion, accumulation, wrapping, uptake, and disruption in these systems allows one to determine the key factors to control the cell-nanomaterial or cell-nanodevice behavior. This knowledge is of fundamental importance for the further progress in nanomedicine, theranostics, oncology, and related fields.

Acknowledgements The part of work related to the carbon nanomaterials was performed in the framework of the research project supported from the Russian Foundation for Basic Research (RFBR), Grant No. 18-29-19198.

\section{References}

1. Shi J, Kantoff PW, Wooster R, Farokhzad OC (2017) Cancer nanomedicine: progress, challenges and opportunities. Nat Rev Cancer 17(1):20-37

2. Lee H, Lee Y, Song C, Cho HR, Ghaffari R, Choi TK, Kim KH, Lee YB, Ling D, Lee H, Yu SJ, Choi SH, Hyeon T, Kim D-H (2015) An endoscope with integrated transparent bioelectronics and theranostic nanoparticles for colon cancer treatment. Nat Commun 6:10059

3. Song S, Faleo G, Yeung R, Kant R, Posselt AM, Desai TA, Tang Q, Roy S (2016) Silicon nanopore membrane (SNM) for islet encapsulation and immunoisolation under convective transport. Sci Rep 6(1):1-9

4. Mikhaylov G, Mikac U, Magaeva AA, Itin VI, Naiden EP, Psakhye I, Babes L, Reinheckel T, Peters C, Zeiser R, Bogyo M, Turk V, Psakhye SG, Turk B, Vasiljeva O (2011) Ferri-liposomes as an MRI-visible drug-delivery system for targeting tumours and their microenvironment. Nat Nanotechnol 6(9):594-602

5. Li D, Zhang Y-T, Yu M, Guo J, Chaudhary D, Wang C-C (2013) Cancer therapy and fluorescence imaging using the active release of doxorubicin from MSPs/Ni-LDH folate targeting nanoparticles. Biomaterials 34(32):7913-7922

6. Lerner MI, Mikhaylov G, Tsukanov AA, Lozhkomoev AS, Gutmanas E, Gotman I, Bratovs A, Turk V, Turk B, Psakhye SG, Vasiljeva O (2018) Crumpled aluminum hydroxide nanostructures as a microenvironment dysregulation agent for cancer treatment. Nano Lett 18(9):5401-5410

7. Gupta A, Landis RF, Li C-H, Schnurr M, Das R, Lee Y-W, Yazdani M, Liu Y, Kozlova A, Rotello VM (2018) Engineered polymer nanoparticles with unprecedented antimicrobial efficacy and therapeutic indices against multidrug-resistant bacteria and biofilms. J Am Chem Soc 140(38):12137-12143

8. Zhou W, Pan T, Cui H, Zhao Z, Chu PK, Yu X-F (2019) Black phosphorus: bioactive nanomaterials with inherent and selective chemotherapeutic effects. Angew Chem (International ed. English) 131(3):779-784

9. Gennis RB (2013) Biomembranes: molecular structure and function. Springer Science \& Business Media, New York

10. Mitaku S (1993) The role of hydrophobic interaction in phase transition and structure formation of lipid membranes and proteins. Phase Transit Multinational J 45(2-3):137-155

11. Thompson RB, Ginzburg VV, Matsen MW, Balazs AC (2001) Predicting the mesophases of copolymer-nanoparticle composites. Science 292(5526):2469-2472 
12. Thompson RB, Ginzburg VV, Matsen MW, Balazs AC (2002) Block copolymer-directed assembly of nanoparticles: forming mesoscopically ordered hybrid materials. Macromolecules 35(3):1060-1071

13. Qian J, Gao H (2010) Soft matrices suppress cooperative behaviors among receptor-ligand bonds in cell adhesion. PLoS ONE 5(8):e12342

14. Gao H, Qian J, Chen B (2011) Probing mechanical principles of focal contacts in cell-matrix adhesion with a coupled stochastic-elastic modelling framework. J R Soc Interface 8(62):12171232. https://doi.org/10.1098/rsif.2011.0157

15. MacKerell AD Jr, Bashford D, Bellott M, Dunbrack RL Jr, Evanseck JD, Field MJ, Fischer S, Gao J, Guo H, Ha S, Joseph-McCarthy D, Kuchnir L, Kuczera K, Lau FTK, Mattos C, Michnick S, Ngo T, Nguyen DT, Prodhom B, Reiher WE, Roux B, Schlenkrich M, Smith JC, Stote R, Straub J, Watanabe M, Wiórkiewicz-Kuczera J, Yin D, Karplus M (1998) All-atom empirical potential for molecular modeling and dynamics studies of proteins. J Phys Chem B 102:3586-3616

16. Monticelli L, Kandasamy SK, Periole X, Larson RG, Tieleman DP, Marrink S-J (2008) The MARTINI coarse-grained force field: extension to proteins. J Chem Theory Comput 4(5):819834

17. Izrailev S, Stepaniants S, Isralewitz B et al (1997) Computational molecular dynamics: challenges, methods, ideas. Springer, Berlin, pp 39-65 (Steered molecular dynamics)

18. Pogodin S, Werner M, Sommer JU, Baulin VA (2012) Nanoparticle-induced permeability of lipid membranes. ACS Nano 6(12):10555-10561

19. Orsi M, Essex JW (2010) Molecular simulations and biomembranes: from biophysics to function. Royal Society of Chemistry, London, pp 76-90 (Passive permeation across lipid bilayers: a literature review)

20. Tsukanov AA, Psakhie SG (2015) A review of computer simulation studies of cell membrane interaction with neutral and charged nano-objects. Quasi-zero-dimensional nanoparticles, drugs and fullerenes. Adv Biomater Dev Med 2(1):44-53

21. Qiao R, Roberts AP, Mount AS, Klaine SJ, Ke PC (2007) Translocation of $\mathrm{C}_{60}$ and its derivatives across a lipid bilayer. Nano Lett 7(3):614-619

22. Wong-Ekkabut J, Baoukina S, Triampo W, Tang I-M, Tieleman DP, Monticelli L (2008) Computer simulation study of fullerene translocation through lipid membrane. Nat Nanotechnol 3:363-368

23. D'Rozario RSG, Wee CL, Wallace EJ, Sansom MSP (2009) The interaction of $\mathrm{C}_{60}$ and its derivatives with a lipid bilayer via molecular dynamics simulations. Nanotechnology 20:115102

24. Jusufi A, DeVane RH, Shinoda W, Klein ML (2011) Nanoscale carbon particles and the stability of lipid bilayers. Soft Matter 7(3):1139-1146

25. Zhang S, Mu Y, Zhang JZH, Xu W (2013) Effect of self-assembly of fullerene nano-particles on lipid membrane. PLoS ONE 8(10):e77436

26. Bozdaganyan ME, Orekhov PS, Shaytan AK, Shaitan K (2014) Comparative computational study of interaction of $\mathrm{C}_{60}$-fullerene and tris-malonyl- $\mathrm{C}_{60}$-fullerene isomers with lipid bilayer: relation to their antioxidant effect. PLoS ONE 9(7):e102487

27. Lai K, Wang B, Zhang Y, Zheng Y (2013) Computer simulation study of nanoparticle interaction with a lipid membrane under mechanical stress. Phys Chem Chem Phys 15(1):270-278

28. Kraszewski S, Tarek M, Treptow W, Ramseyer C (2010) Affinity of $\mathrm{C}_{60}$ neat fullerenes with membrane proteins: a computational study on potassium channels. ACS Nano 4:4158-4164

29. Monticelli L, Barnoud J, Orlowski A, Vattulainen I (2012) Interaction of $\mathrm{C}_{70}$ fullerene with the Kv1.2 potassium channel. Phys Chem Chem Phys 14(36):12526-12533

30. Kraszewski S, Tarek M, Ramseyer C (2011) Uptake and translocation mechanisms of cationic amino derivatives functionalized on pristine $\mathrm{C}_{60}$ by lipid membranes: a molecular dynamics simulation study. ACS Nano 5:8571-8578

31. Tieleman DP (2006) Computer simulations of transport through membranes: passive diffusion, pores, channels and transporters. Proc Aust Physiol Soc 37:15-27 
32. Monticelli L, Salonen E, Ke PC, Vattulainen I (2009) Effects of carbon nanoparticles on lipid membranes: a molecular simulation perspective. Soft Matter 5(22):4433-4445

33. Makarucha AJ, Todorova N, Yarovsky I (2011) Nanomaterials in biological environment: a review of computer modelling studies. Eur Biophys J 40:103-115

34. Esfand R, Tomalia DA (2001) Poly(amidoamine) (PAMAM) dendrimers: from biomimicry to drug delivery and biomedical applications. Drug Discov Today 6(8):427-436

35. Kolotylo M, Holovatiuk V, Bondareva J, Lukin O, Rozhkov V (2019) Synthesis of sulfonimidebased dendrimers and dendrons possessing mixed $1 \rightarrow 2$ and $1 \rightarrow 4$ branching motifs. Tetrahedron Lett 60(4):352-354

36. Rozhkov VV, Kolotylo MV, Onys'ko PP, Lukin O (2016) Synthesis of sulfonimide-based branched arylsulfonyl chlorides. Tetrahedron Lett 57(3):308-309

37. Maiti PK, Çağın T, Wang G, Goddard WA (2004) Structure of PAMAM dendrimers: generations 1 through 11. Macromolecules 37(16):6236-6254

38. Lee H, Larson RG (2006) Molecular dynamics simulations of PAMAM dendrimer-induced pore formation in DPPC bilayers with a coarse-grained model. J Phys Chem B 110:18204-18211

39. Lee H, Larson RG (2008) Coarse-grained molecular dynamics studies of the concentration and size dependence of fifth- and seventh-generation PAMAM dendrimers on pore formation in DMPC bilayer. J Phys Chem B 112:7778-7784

40. Lee H, Larson RG (2008) Lipid bilayer curvature and pore formation induced by charged linear polymers and dendrimers: the effect of molecular shape. J Phys Chem B 112:12279-12285

41. Kelly CV, Leroueil PR, Nett EK, Wereszczynski JM, Baker JR, Orr BG, Banaszak Holl MM, Andricioaei I (2008) Poly(amidoamine) dendrimers on lipid bilayers I: free energy and conformation of binding. J Phys Chem B 112(31):9337-9345

42. Kelly CV, Leroueil PR, Orr BG, Banaszak Holl MM, Andricioaei I (2008) Poly(amidoamine) dendrimers on lipid bilayers II: effects of bilayer phase and dendrimer termination. J Phys Chem B 112:9346-9353

43. Yan LT, Yu X (2009) Enhanced permeability of charged dendrimers across tense lipid bilayer membranes. ACS Nano 3(8):2171-2176

44. Ting CL, Wang ZG (2011) Interactions of a charged nanoparticle with a lipid membrane: implications for gene delivery. Biophys J 100(5):1288-1297

45. Ginzburg VV, Balijepalli S (2007) Modeling the thermodynamics of the interaction of nanoparticles with cell membranes. Nano Lett 7(12):3716-3722

46. Li Y, Chen X, Gu N (2008) Computational investigation of interaction between nanoparticles and membranes: hydrophobic/hydrophilic effect. J Phys Chem B 112(51):16647-16653

47. Alexeev A, Uspal WE, Balazs AC (2008) Harnessing Janus nanoparticles to create controllable pores in membranes. ACS Nano 2(6):1117-1122

48. Yang K, Ma YQ (2010) Computer simulation of the translocation of nanoparticles with different shapes across a lipid bilayer. Nat Nanotechnol 5(8):579-583

49. Lin J, Zhang H, Chen Z, Zheng Y (2010) Penetration of lipid membranes by gold nanoparticles: insights into cellular uptake, cytotoxicity, and their relationship. ACS Nano 4(9):5421-5429

50. Guo R, Mao J, Yan LT (2013) Unique dynamical approach of fully wrapping dendrimer-like soft nanoparticles by lipid bilayer membrane. ACS Nano 7(12):10646-10653

51. Carr R, Weinstock IA, Sivaprasadarao A, Müller A, Aksimentiev A (2008) Synthetic ion channels via self-assembly: a route for embedding porous polyoxometalate nanocapsules in lipid bilayer membranes. Nano Lett 8(11):3916-3921

52. Dykman L, Khlebtsov N (2012) Gold nanoparticles in biomedical applications: recent advances and perspectives. Chem Soc Rev 41:2256-2282

53. Nangia S, Sureshkumar R (2012) Effects of nanoparticle charge and shape anisotropy on translocation through cell membranes. Langmuir 28(51):17666-17671

54. Tsukanov AA, Pervikov AV, Lozhkomoev AS (2020) Bimetallic Ag-Cu nanoparticles interaction with lipid and lipopolysaccharide membranes. Comput Mater Sci 173:109396

55. Daw MS, Baskes MI (1984) Embedded-atom method: derivation and application to impurities, surfaces, and other defects in metals. Phys Rev B 29(12):6443 
56. Williams PL, Mishin Y, Hamilton JC (2006) An embedded-atom potential for the $\mathrm{Cu}-\mathrm{Ag}$ system. Modell Simul Mater Sci Eng 14(5):817

57. Hohenberg P, Kohn W (1964) Inhomogeneous electron gas. Phys Rev 136:B864

58. Kohn W, Sham LJ (1965) Self-consistent equations including exchange and correlation effects. Phys Rev 140(4A):A1133

59. Bekyarova E, Ni Y, Malarkey EB, Montana V, McWilliams JL, Haddon RC, Parpura V (2005) Applications of carbon nanotubes in biotechnology and biomedicine. J Biomed Nanotechnol 1(1):3-17

60. Rotkin SV (2010) Electronic properties of nonideal nanotube materials: helical symmetry breaking in DNA hybrids. Annu Rev Phys Chem 61:241-261

61. Tsukanov AA, Grachev EA, Rotkin SV (2007) Modeling of the SWNT-DNA complexes in the water solution. APS March meeting, American Physical Society, 5-9 Mar 2007, abstract id. V28.002

62. Lopez CF, Nielsen SO, Moore PB, Klein ML (2004) Understanding nature's design for a nanosyringe. PNAS Proc Nat Acad Sci U S A 101:4431-4434

63. Zimmerli U, Koumoutsakos P (2008) Simulations of electrophoretic RNA transport through transmembrane carbon nanotubes. Biophys J 94(7):2546-2557

64. Höfinger S, Melle-Franco M, Gallo T, Cantelli A, Calvaresi M, Gomes JANF, Zerbetto F (2011) A computational analysis of the insertion of carbon nanotubes into cellular membranes. Biomaterials 32:7079-7085

65. Shi XH, Kong Y, Gao HJ (2008) Coarse grained molecular dynamics and theoretical studies of carbon nanotubes entering cell membrane. Acta Mech Sin 24:161-169

66. Pogodin S, Baulin VA (2010) Can a carbon nanotube pierce through a phospholipid bilayer? ACS Nano 4:5293-5300

67. Ben-Shaul A, Szleifer I, Gelbart WM (1985) Chain organization and thermodynamics in micelles and bilayers. AIP J Chem Phys 83(7):3597-3611

68. Pogodin S, Slater NKH, Baulin VA (2011) Surface patterning of carbon nanotubes can enhance their penetration through a phospholipid bilayer. ACS Nano 5:1141-1146

69. Gangupomu VK, Capaldi FM (2011) Interactions of carbon nanotube with lipid bilayer membranes. J Nanomater 2011:830436

70. Shityakov S, Dandekar T (2011) Molecular dynamics simulation of POPC and POPE lipid membrane bilayers enforced by an intercalated single-wall carbon nanotube. NANO 6(01):1929

71. Parthasarathi R, Tummala NR, Striolo A (2012) Embedded single-walled carbon nanotubes locally perturb DOPC phospholipid bilayers. J Phys Chem B 116:12769-12782

72. Kraszewski S, Bianco A, Tarek M, Remaseyer C (2012) Insertion of short amino-functionalized single-walled carbon nanotubes into phospholipid bilayer occurs by passive diffusion. PLoS ONE 7(7):e40703

73. Raczyński P, Górny K, Pabiszczak M, Gburski Z (2013) Nanoindentation of biomembrane by carbon nanotubes-MD simulation. Comput Mater Sci 70:13-18

74. Mousavi SZ, Amjad-Iranagh S, Nademi Y, Modarress H (2013) Carbon nanotube-encapsulated drug penetration through the cell membrane: an investigation based on steered molecular dynamics simulation. J Membr Biol 246(9):697-704

75. Baoukina S, Monticelli L, Tieleman DP (2013) Interaction of pristine and functionalized carbon nanotubes with lipid membranes. J Phys Chem B 117:12113-12123

76. Thomas M, Enciso M, Hilder TA (2015) Insertion mechanism and stability of boron nitride nanotubes in lipid bilayers. J Phys Chem B 119(15):4929-4936

77. Tsukanov AA, Psakhie SG (2016) Potential of mean force analysis of short boron nitride and carbon nanotubes insertion into cell membranes. Adv Biomater Dev Med 3(1):1-9

78. Won CY, Aluru NR (2008) Structure and dynamics of water confined in a boron nitride nanotube. J Phys Chem C 112(6): 1812-1818

79. Alshehri MH (2018) Interactions of boron nitride nanotubes with lipid bilayer membranes. $\mathbf{J}$ Comput Theor Nanosci 15(1):311-316 
80. Titov AV, Kral P, Pearson R (2010) Sandwiched graphene-membrane superstructures. ACS Nano 4:229-234

81. Guo R, Mao J, Yan L-T (2013) Computer simulation of cell entry of graphene nanosheet. Biomaterials 34(17):4296-4301

82. Wang J, Wei Y, Shi X, Gao H (2013) Cellular entry of graphene nanosheets: the role of thickness, oxidation and surface adsorption. RSC Adv 3(36):15776-15782

83. Li Y, Yuan H, von dem Bussche A, Creighton M, Hurt RH, Kane AB, Gao H (2013) Graphene microsheets enter cells through spontaneous membrane penetration at edge asperities and corner sites. PNAS Proc Nat Acad Sci U S A 110(30):12295-12300

84. Mao J, Guo R, Yan L-T (2014) Simulation and analysis of cellular internalization pathways and membrane perturbation for graphene nanosheets. Biomaterials 35(23):6069-6077

85. Tu Y, Lv M, Xiu P, Huynh T, Zhang M, Castelli M, Liu Z, Huang Q, Fan C, Fang H, Zhou R (2013) Destructive extraction of phospholipids from Escherichia coli membranes by graphene nanosheets. Nat Nanotechnol 8(8):594-601

86. Tsukanov AA, Psakhie SG (2016) Adsorption of charged protein residues on an inorganic nanosheet: computer simulation of LDH interaction with ion channel. AIP Conf Proc 1760(1):020066

87. Shroll RM, Straatsma TP (2003) Molecular basis for microbial adhesion to geochemical surfaces: computer simulation of Pseudomonas aeruginosa adhesion to goethite. Biophys $\mathrm{J}$ 84(3): 1765-1772

Open Access This chapter is licensed under the terms of the Creative Commons Attribution 4.0 International License (http://creativecommons.org/licenses/by/4.0/), which permits use, sharing, adaptation, distribution and reproduction in any medium or format, as long as you give appropriate credit to the original author(s) and the source, provide a link to the Creative Commons license and indicate if changes were made.

The images or other third party material in this chapter are included in the chapter's Creative Commons license, unless indicated otherwise in a credit line to the material. If material is not included in the chapter's Creative Commons license and your intended use is not permitted by statutory regulation or exceeds the permitted use, you will need to obtain permission directly from the copyright holder.

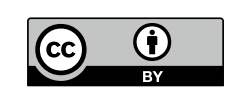

